\title{
Immunization with UV-Induced Apoptotic Cells Generates Monoclonal Antibodies Against Proteins Differentially Expressed in Hepatocellular Carcinoma Cell Lines
}

\author{
HILAL CELIKKAYA, ${ }^{1,3}$ CEREN CIRACI,,${ }^{1,4}$ EMIN OZTAS, ${ }^{1,2}$ M. ENDER AVCI, ${ }^{1}$ \\ MEHMET OZTURK, ${ }^{1}$ and TAMER YAGCI ${ }^{1}$
}

\begin{abstract}
Early and differential diagnosis of hepatocellular carcinoma (HCC) requires sensitive and specific tissue and serum markers. On the other hand, proteins involved in tumorigenesis are extensively modulated on exposure to apoptotic stimuli, including ultraviolet (UVC) irradiation. Hence, we generated monoclonal antibodies by using UVC-irradiated apoptotic cells of an HCC cell line, HUH7, aiming to explore proteins differentially expressed in tumors and apoptosis. We obtained 18 hybridoma clones recognizing protein targets in apoptotic HUH7 cells, and clone 6D5 was chosen for characterization studies because of its strong reactivity in cell-ELISA assay. Subtype of the antibody was IgG3 $(\kappa)$. Targets of $6 \mathrm{D5}$ antibody were found to be abundantly expressed in all HCC cell lines except FLC4, which resembles normal hepatocytes. We also observed the secretion of 6D5 ligands by some of the HCC cell lines. Moreover, cellular proteins recognized by the antibody displayed a late upregulation in UVC-induced apoptotic cells. We concluded that 6D5 target proteins are modulated in liver tumorigenesis and apoptotic processes. We therefore propose the validation of our antibody in tissue and serum samples of HCC patients to assess its potential use for the early diagnosis of HCC and to understand the role of $6 \mathrm{D5}$ ligands in liver carcinogenesis.
\end{abstract}

\section{INTRODUCTION}

$\mathbf{H}$ EPATOCELLULAR CARCINOMA (HCC) is one of the most frequently occurring cancers worldwide, and in some regions of Asia, represents the primary cause of death due to cancer. ${ }^{(1,2)}$ Its incidence continues to increase throughout the world due to hepatitis B virus (HBV) infections in developing countries, ${ }^{(3)}$ and hepatitis $\mathrm{C}$ virus $(\mathrm{HCV})$ infections ${ }^{(4)}$ and excessive alcohol intake ${ }^{(5)}$ in developed western countries and Japan. These liver-damaging agents lead to liver cirrhosis, which, once established, constitutes the major background for HCC development. ${ }^{(6)}$ Therefore, patients with liver cirrhosis are periodically surveyed for the diagnosis of HCC at early stages of tumor development. Serum $\alpha$-fetoprotein levels (AFP) and hepatic ultrasonography (HUS) are the screening tools of choice, ${ }^{(7)}$ yet other promising biomarkers, such as des-gamma carboxypro- thrombin, lens culinaris-agglutinin reactive AFP, human hepatocyte growth factor-1 (HGF-1), insulin-like growth factor-1 (IGF-1), and glypican-3, are currently under intensive investigation. ${ }^{(8,9)}$ However, the sensitivity and the specificity of these tumor markers for HCC are variable and most of them are not introduced yet into routine clinical practice. ${ }^{(9)}$ Therefore, the development of novel markers for HCC with stronger sensitivity and specificity is of great importance for the surveillance of patients with liver cancer risk.

Cancer cells develop various strategies, including resistance to apoptotic stimuli, in order to escape from host immune surveillance mechanisms. ${ }^{(10)}$ They increase the expression of antiapoptotic proteins, while decreasing proapoptotic ones, either by mutations or epigenetic regulations. ${ }^{(11,12)}$ In order to explore proteins that are modulated in apoptosis and tumorigenesis processes, we produced monoclonal antibodies by using as im-

\footnotetext{
${ }^{1}$ Department of Molecular Biology and Genetics, Bilkent University, Ankara, Turkey.

${ }^{2}$ Department of Medical Histology, Gulhane Military Medical Academy, Ankara, Turkey.

${ }^{3}$ Current address: Department of Cellular and Molecular Pharmacology, The Cancer Institute of New Jersey, The Robert Wood Johnson Medical School, University of Medicine and Dentistry of New Jersey, New Brunswick, New Jersey.

${ }^{4}$ Current address: Department of Biochemistry, Biophysics and Molecular Biology, Iowa State University, Ames, Iowa.
} 
munogen UVC-induced apoptotic cells of an HCC cell line, HUH7. The expression of the targets of clone 6D5 was studied in both HCC cell lines and apoptosis-induced HUH7 and Jurkat cells.

\section{MATERIALS AND METHODS}

\section{HCC cell lines and antibodies}

HCC-derived HUH7, Hep40, Hep3B, Hep3B-TR, PLC/PRF5, FLC4, SK-Hep1, Focus, Mahlavu, SNU182, SNU387, SNU398, SNU449, and SNU475 and hepatoblastoma-derived HepG2 cell lines were analyzed. SNU182, SNU387, SNU398, SNU449, and SNU475 cell lines were grown in RPMI-1640 medium supplemented with $10 \%$ fetal bovine serum (FBS) $100 \mathrm{IU}$ penicillin, $100 \mu \mathrm{g}$ streptomycin, and nonessential amino acids (RPMI-10). All other cell lines were grown in Dulbecco's modified Eagle's medium (DMEM) supplemented with $10 \%$ of FBS, 100 IU penicillin, $100 \mu \mathrm{g}$ streptomycin, and nonessential amino acids (DMEM-10). Sp2/0 mouse myeloma cells were grown in high-glucose DMEM-10 and used as fusion partner for hybridoma production. A T-cell leukemia cell line, Jurkat, was grown in RPMI-10. Anticalnexin monoclonal antibody was a kind gift of Dr. Mehmet Ozturk (Bilkent University, Ankara, Turkey). Anti-Fas antibody (clone CH11) was purchased from Upstate Biotechnology (Lake Placid, NY).

\section{Apoptosis induction in HUH7 and Jurkat cells}

Two well-established apoptosis-induction methods, UVC irradiation and anti-Fas treatment, were used for $\mathrm{HUH7}$ and $\mathrm{Ju}-$ rkat cells, respectively. ${ }^{(13)}$ Briefly, HUH7 cells were grown to $80-90 \%$ confluency in $10 \mathrm{~cm}$ tissue culture plates. Cells were washed three times with phosphate-buffered saline (PBS) and exposed to UVC irradiation $\left(20 \mathrm{~J} / \mathrm{M}^{2}\right)$ in UV-crosslinker apparatus (Stratagene, La Jolla, CA). After the addition of fresh medium, plates were transferred into $\mathrm{CO}_{2}$ incubator. Jurkat cells were plated at a density of $5 \times 10^{5} / \mathrm{mL}$ and treated for $24 \mathrm{~h}$ with $50 \mathrm{ng} / \mathrm{mL}$ of anti-Fas antibody. Both treated and untreated HUH7 and Jurkat cells were then collected at desired time points for subsequent apoptosis detection and Western blot assays. Apoptosis was analyzed both by examining morphologic changes under the light microscope and by Annexin V staining (Annexin V-FITC Apoptosis Detection Kit, Sigma-Aldrich, St. Louis, MO) of apoptotic cells, according to the manufacturer's instructions.

\section{Immunization and production of monoclonal antibodies}

5-10 × $10^{6}$ UVC-treated apoptotic HUH7 cells were scraped from culture plates and, after washing with PBS, were injected into the peritoneal cavity of 6- to 8-week-old BALB/c mice (day 0). Three more injections were repeated at 3 week intervals, and immunization efficiency was assessed on days 25 and 45 by cell-ELISA, as described below. Three days after the last injection, immunized mice were sacrificed and their spleen cells were fused with $\mathrm{Sp} 2 / 0$ mouse myeloma cells by standard protocol. ${ }^{(14)}$ Hybrid cells were plated into 96-well tissue culture plates in hybridoma growth medium consisting of high-glucose
DMEM supplemented with 20\% FCS, 100 IU penicillin, 100 $\mu \mathrm{g}$ streptomycin, nonessential amino acids, and hybridoma selection reagents (hypoxantine, aminopterin, thymidine (HAT); Sigma-Aldrich). Screening of hybrid cells was performed by cell-ELISA and clones with high absorbance values were subjected to subclonings by limited dilution. Isotype of antibodies was determined by ImmunoPure Monoclonal Antibody Isotyping Kit (Pierce, Rockford, IL), according to the manufacturer's instructions.

\section{Cell-ELISA}

HUH7 cells were plated in 96-well tissue culture plates at a density of $5 \times 10^{4}$ cells/well, and UVC irradiation was applied when cells reached confluency. After 16 h, medium was aspirated from the wells of culture plates and cells were fixed by $100 \%$ ice-cold methanol. Next, fixed cells were incubated at room temperature with supernatant of growing hybridomas for $2 \mathrm{~h}$ followed by incubation for $1 \mathrm{~h}$ with alkaline phosphatase (AP) conjugated anti-mouse IgG (Sigma-Aldrich) at 1:1000 dilution. Colorimetric reaction was allowed to occur by using paranitrophenylphosphate (pNPP) substrate solution (pNPP tablets, Sigma-Aldrich). Plates were read at $\mathrm{A}_{405}$ using a microplate reader (Beckman, Fullerton, CA). Between each step of cell-ELISA, wells were washed gently with PBS to prevent the detachment of fixed cells.

\section{Western blotting}

Total cell lysates from $14 \mathrm{HCC}$ and Jurkat cell lines were prepared in NP-40 lysis buffer (50 mM Tris-HCl pH 8.0, 150 $\mathrm{mM} \mathrm{NaCl}, 1 \%$ Non-idet P40 [v/v] and a cocktail of EDTA-free protease inhibitors [Roche Diagnostics, Mannheim, Germany]). Protein content was measured by Bradford assay. ${ }^{(15)}$ Equalized lysates were run on $10 \%$ SDS-PAGE and then transferred onto polyvinylidene fluoride (PVDF) membranes by using semi-dry transfer apparatus (Bio-Rad Laboratories, Hercules, CA). Membranes were blocked overnight with $5 \%$ dry milk in Trisbuffered saline (TBS) containing 0.1\% Tween-20 (TBS-T). Undiluted 6D5 hybridoma supernatant was used as primary antibody. After washing three times with TBS-T, horseradish peroxidase (HRP) conjugated anti-mouse IgG (Sigma-Aldrich) was used as secondary antibody at 1:5000 dilution. Protein bands were visualized using ECL Plus chemiluminescent substrate (Amersham, Piscataway, NJ). For equal loading control, membranes were reprobed with anticalnexin antibody.

\section{Immunofluorescence}

HUH7, HepG2, and PLC/PRF5 cells were grown on cover slips in 6-well plates. PBS was used in all washing steps. Cells were fixed in $2 \%$ paraformaldehyde in PBS and permeabilized in PBS containing $0.2 \%$ triton X-100. After blocking with $2 \%$ bovine serum albumin (BSA) in PBS, cells were incubated for $2 \mathrm{~h}$ at room temperature with undiluted 6D5 hybridoma supernatant. Fluorescein isothiocyanate (FITC) conjugated antimouse IgG (Sigma-Aldrich) was used as secondary antibody at 1:200 dilution. Nuclei counterstaining was performed with $4^{\prime}, 6-$ diamidino-2-phenylindole (DAPI); cover slips were mounted on glass slides and examined under fluorescent microscope (Carl Zeiss, Göttingen, Germany). Merged images were produced by using AxioVision image processing software (Carl Zeiss). 


\section{Immunoprecipitation and Western blotting (IP-WB) of secreted proteins}

First serum-free culture media of HCC cell lines were incubated for $2 \mathrm{~h}$ at $4^{\circ} \mathrm{C}$ with $100 \mu \mathrm{L}$ of $6 \mathrm{D} 5$ hybridoma supernatant; then $50 \mu \mathrm{L}$ of protein $\mathrm{G}$ agarose beads (East Coast Biologicals), pre-cleaned with three PBS washes, were added. The mixture was incubated overnight at $4^{\circ} \mathrm{C}$ with continuous rotation. Next, beads were precipitated by centrifugation, washed three times with PBS, and antigen-antibody complexes were eluted by resuspending beads with an equal volume of $2 \mathrm{X}$ SDSPAGE sample loading buffer (100 mM Tris-Cl pH 6.8, 4\% SDS, $0.2 \%$ bromophenol blue, $20 \%$ glycerol and freshly added 200 mM DTT). SDS-PAGE and Western blotting were performed as described above.

\section{RESULTS}

\section{Immunization with apoptotic HUH7 cells}

To determine the appropriate time for harvesting cells following apoptosis induction, we looked for the presence of apoptotic cells at various times after ultraviolet irradiation. HUH7 cells were grown on cover slips and exposed to UVC at 20 $\mathrm{J} / \mathrm{M}^{2}$. FITC-labeled Annexin V analyses of UVC-treated and untreated $\mathrm{HUH} 7$ cells were performed at $4 \mathrm{~h}$ and $24 \mathrm{~h}$ postUVC treatment. Treated cells displayed strong reactivity with FITC-labeled Annexin V at both time points, while very low staining, if any, was observed in unstimulated HUH7 cells (Fig. 1). Therefore, we started immunization of BALB/c mice with cells harvested at $16 \mathrm{~h}$ post-UVC treatment, and we repeated injections of apoptotic cells at 3 week intervals. The development of an immune response in mice sera was assessed by cellELISA, as described in the above Materials and Methods section (data not shown).

\section{Production of monoclonal antibodies}

Following the fusion of spleen cells of immunized mice with Sp2/0 myeloma cells, cell-ELISA assays were performed. This time HUH7 cells were grown to confluency in 96-well tissue culture plates and then exposed to UVC to screen apoptotic cells for their immunoreactivity with antibodies secreted by hybridoma clones. We obtained 18 hybridoma clones recognizing protein targets in apoptotic cells. Out of these, clones 6D5, 6E10, 9C11, and 11G8 displayed higher cell-ELISA absorbance values, with 6D5 showing highest ELISA titer (data not shown). All reactive clones were subcloned two times by limiting dilution, and monoclonal antibody-secreting hybridomas were expanded in culture for supernatant collection and cryopreservation. Characterization studies were then initiated with 6D5 antibody by reasoning that high cell-ELISA titer of this clone might correlate with overexpression of its ligands in both HCC cell lines and cells undergoing apoptosis upon ultraviolet irradiation. The isotype of the antibody was found to be $\operatorname{IgG} 3(\kappa)$.

\section{$6 D 5$ recognizes differentially expressed proteins in HCC cell lines}

We first analyzed the immunoreactivity of 6D5 antibody with whole cell lysates of HCC cell lines. In three separate Western blot assays, two major proteins with closer molecular weights

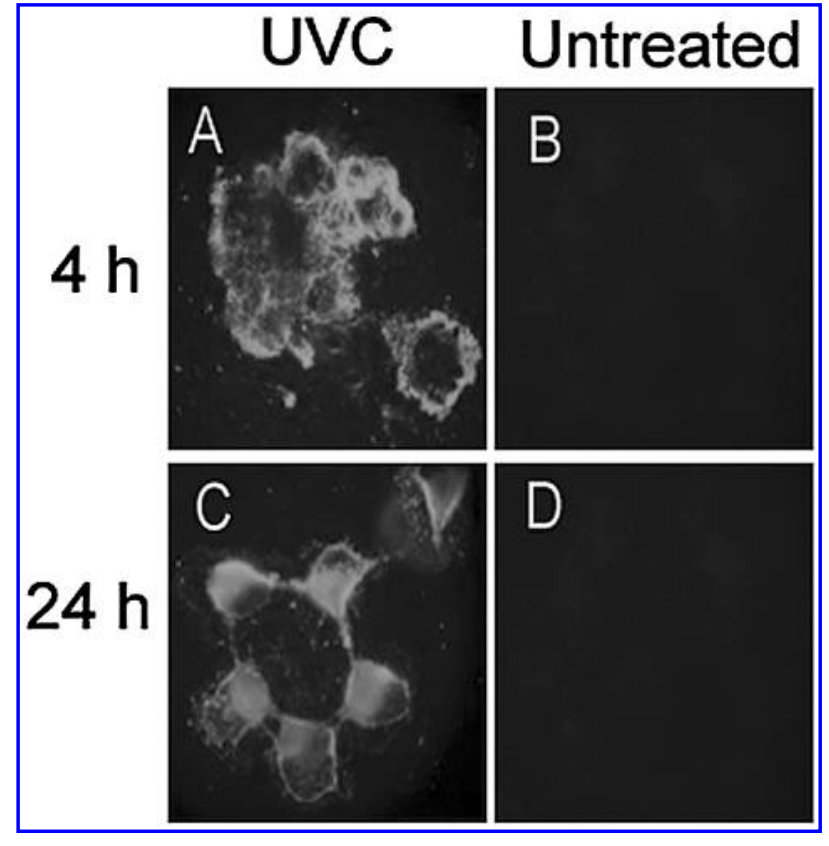

FIG. 1. Annexin V-FITC staining of HUH7 cells after UVC irradiation. Cells were grown on cover slips in $10 \mathrm{~cm}$ culture plates and then exposed to $20 \mathrm{~J} / \mathrm{cm}^{2}$ of UVC. Annexin V-FITC staining was performed at $4 \mathrm{~h}(\mathbf{A})$ and $24 \mathrm{~h}(\mathbf{C})$ post-UVC treatment. (B and D) Untreated controls of $\mathbf{A}$ and $\mathbf{C}$, respectively.

of $\sim 80 \mathrm{kDa}$ were found to be expressed either alone or concomitantly in all studied cells (Fig. 2, upper row). Strong band intensity suggested an abundant expression of the proteins in these cell lines. Equal protein loading analysis was performed by reprobing the membrane with anticalnexin antibody (Fig. 2, lower row). Lysates of all cells have been shown to contain equal amounts of total protein, except SK-Hep1 cells, which displayed a significant decrease in calnexin protein level. This result repeatedly persisted in independent immunoblots, suggesting a downregulation of this ER chaperone in that cell line. Cell lines deriving from undifferentiated and invasive tumors, including SNU475, SNU398, SNU449, SNU387, SK-Hep1, and Mahlavu, seemed to express 6D5 ligands stronger than did differentiated HUH7 and HepG2 cell lines (Fig. 2, left and middle panels). However, no other correlation could be established between the protein expression patterns and phenotypic characteristics of cell lines. Protein expression was also examined in two isogenic cell lines, Hep3B and Hep3B-TR, and a third protein band with lowest molecular weight was observed only in Hep3B cells (Fig. 2, right panel). These cell lines have been described elsewhere, and Hep3B-TR cells differ from Hep3B cells by their resistance to the growth inhibitory effect of TGFbeta in cell culture. ${ }^{(16)} \mathrm{We}$ also compared target protein expression in HUH7 and FLC4 cells and found that the latter was totally devoid of any 6D5 ligands (Fig. 3). The hepatoma cell line FLC4 was established from a well-differentiated HCC and found to possess normal liver functions. ${ }^{(17,18)}$ We therefore suggest that 6D5 targets are upregulated in HCCs.

\section{$6 D 5$ stained the cytoplasm of HCC cell lines}

Next we visualized the cellular localization of 6D5 targets by immunofluorescence microscopy in three HCC cell lines. 


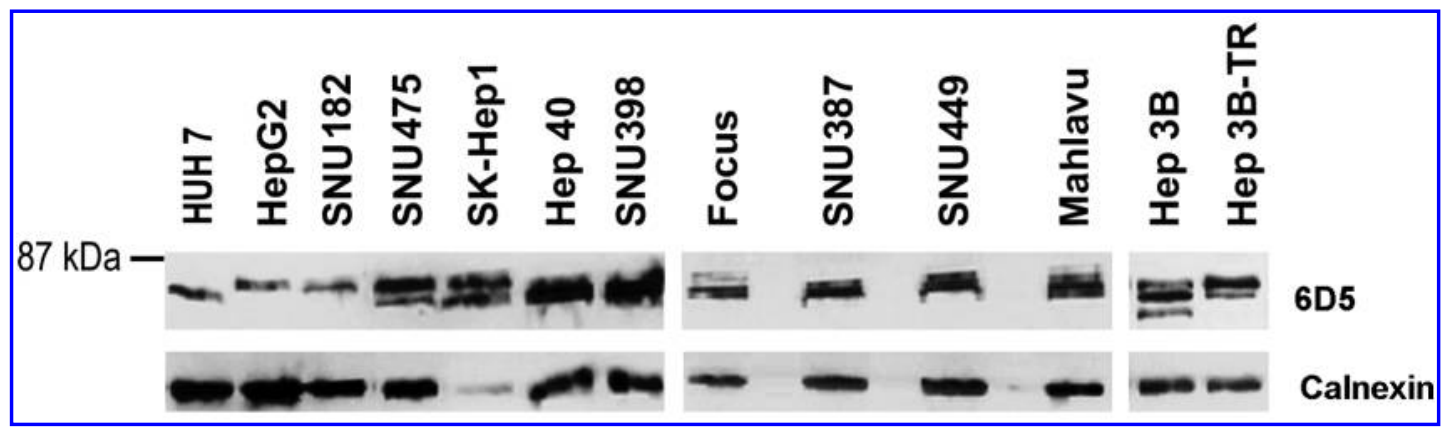

FIG. 2. Expression of 6D5 target proteins in HCC cell lines. Whole cell lysates of HCC cell lines were analyzed in three separate Western blots. Total protein extracts on PVDF membranes were first probed with 6D5 (upper row) and then with anticalnexin (lower row) antibodies. Two major protein bands with molecular weight close to $80 \mathrm{kDa}$ were displayed by all cells alone (HUH7, HepG2, SNU-182, Hep40, SNU-398, SNU-387) or concomitantly (SNU-475, SK-Hep1, Focus, SNU-449, Mahlavu, Hep3B-TR). Only Hep3B cells expressed a third protein band with the lowest molecular weight.

6D5 stained the cytoplasm of HUH7, HepG2, and PLC/PRF5 cells. The strong intensity of the fluorescent signal supported our observation that proteins recognized by this antibody are abundantly expressed in HCC cell lines (Fig. 4).

\section{Secretion of $6 D 5$ targets form HCC cells}

Protein secretion was searched first in the supernatant of isogenic cell lines Hep3B and Hep3B-TR. Cells were cultured up to $70-80 \%$ confluency and then starved for $48 \mathrm{~h}$ before the collection of supernatants. This strategy allowed us to eliminate abundant proteins deriving from fetal calf serum. In the IP-WB experiment, we detected expression of target proteins only in the Hep3B culture medium (Fig. 5). A lower band with sharp intensity and a heavier weak one were observed. However, the third protein band detected in whole cell lysate of Hep3B cells (Fig. 2) was absent in IP-WB (Fig. 5). The differential behavior of Hep3B and Hep3B-TR cell lines in protein secretion also supports our finding that target proteins are differentially expressed in these cells (Fig. 2). We also analyzed the secretion of target proteins from other HCC cell lines, including Focus, HUH7, SNU398, and Mahlavu. Following IP-WB with their

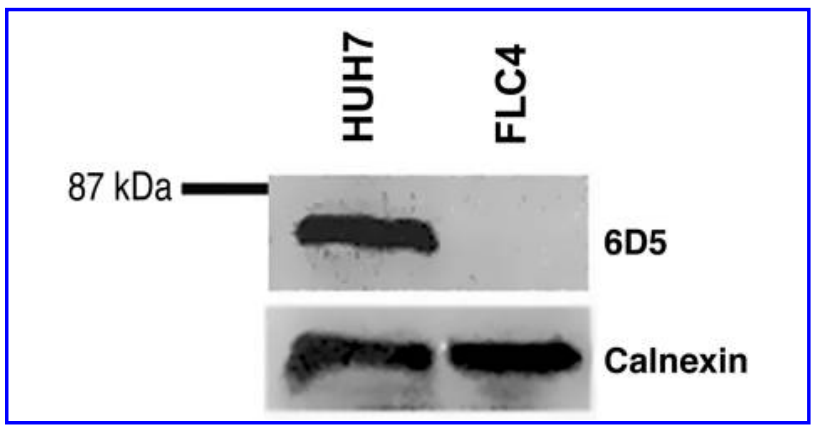

FIG. 3. Lack of target protein expression in FLC4 cell line. Target protein expression of HUH7 cells and FLC4 cells was compared by Western blotting. Immunoblot was first probed with 6D5 antibody. No protein expression was observed in FLC4 cells (upper row). Equal protein loading was controlled by reprobing the membrane with anti-calnexin antibody (lower row). supernatants, we detected faint protein bands in the supernatants of Focus and SNU398 cells, but not in those of Mahlavu and HUH7 cells (data not shown).

\section{Effects of apoptosis induction on the expression of 6D5 targets}

Next, we examined the modulation of 6D5 ligands in Jurkat and HUH7 cells treated with anti-Fas antibody and UVC, respectively. Both cells were collected at $24 \mathrm{~h}$ post-treatment, and the expression of 6D5 target proteins was visualized by Western blotting. An induction on protein level was observed for UVC-treated HUH7 cells, but unexpectedly neither untreated nor anti-Fas-treated Jurkat cells displayed 6D5 ligand expression (Fig. 6, upper row). The same membrane was then reprobed with 9C11 antibody, and target proteins of this clone were found to be expressed by both cell lines, in both apoptotic and nonapoptotic conditions. We therefore turned to investigate in more detail the modulation of 6D5 proteins in HUH7 cells committed to apoptosis under UVC treatment. We harvested cells at various time points after UVC irradiation and compared the protein expression with that of untreated HUH7 cells. As shown in Figure 7, protein expression was significantly reduced during the initial phases of apoptosis (2, 4, and $8 \mathrm{~h}$ after treatment), followed by a recovery at hour 16 , with protein levels exceeding those of untreated cells. This recovery was sustained at hour 24 post-UVC treatment, indicating an intermediate-to-late upregulation of 6D5 ligands in cells undergoing apoptosis.

\section{DISCUSSION}

The choice of HUH7 cells as immunogen is based on our previous observations that these cells remain attached to culture plate surfaces even after they commit suicide. ${ }^{(13)}$ In fact, this property facilitated the collection of cells for immunization with minimal cell loss and allowed us to perform accurate cellELISA assays. On the other hand, HUH7 cells consist of heterogeneous cell populations, which make them an ideal source for the identification of novel markers for liver tumors, as well as the characterization of liver precursor cells involved in hepatocellular carcinogenesis. ${ }^{(19)}$ 


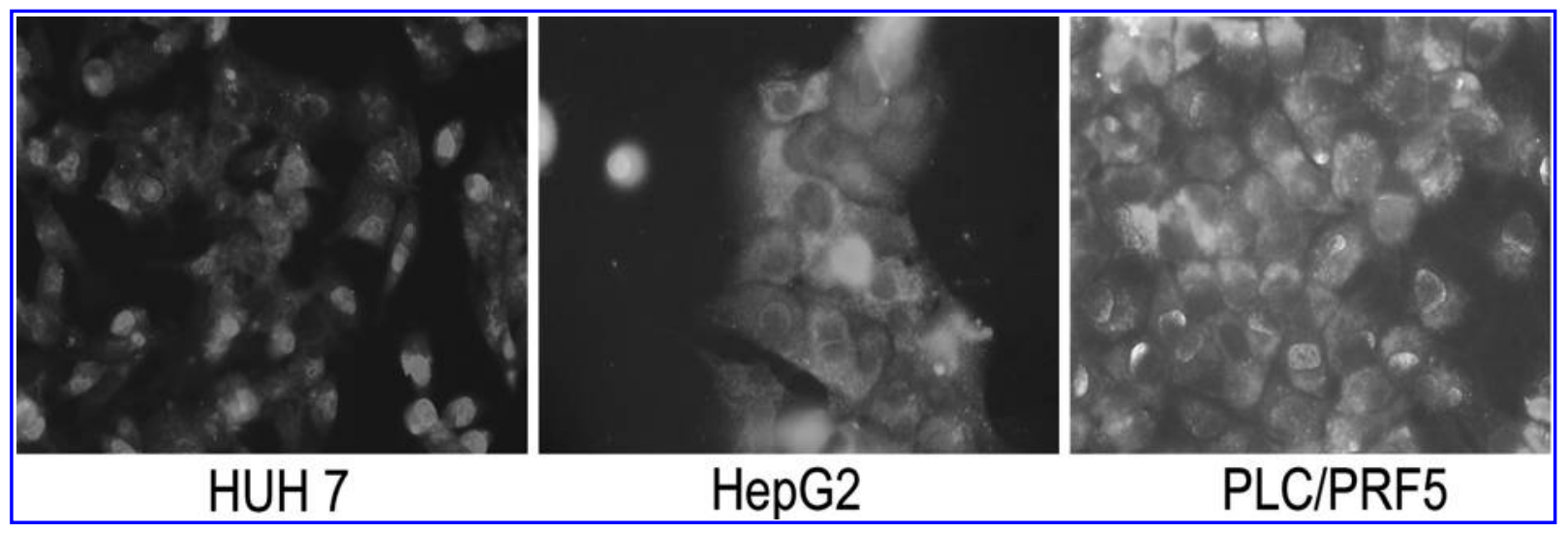

FIG. 4. Immunofluorescence analysis of HCC cell lines with 6D5 antibody. Cells were grown on cover slips and cellular location of target proteins in HUH7, HepG2, and PLC/PRF5 cell lines was evaluated by indirect immunofluorescence using 6D5 antibody. DAPI was used for nuclei counterstaining, and merged images were obtained by using image processing software. All cell lines exhibited strong cytoplasmic staining.

Our Western blot analyses pointed out several important conclusions. First, proteins recognized by 6D5 antibody are abundantly and differentially expressed in HCC cell lines, and this expression appears to increase in poorly differentiated cells. Second, target proteins are differentially expressed in both cell lysates and supernatants of Hep3B and Hep3B-TR isogenic cell lines. The fact that these cells mainly differ in their response to TGF- $\beta$ suggests a modulation of 6D5 protein expression by this cytokine-mediated pathway. Third, only FLC4 cell line failed to express proteins recognized by our antibody. Well-differentiated FLC4 cells are known to have relatively well-preserved liver cell functions, such as albumin synthesis and enzyme and drug metabolism activities. ${ }^{(20,21)}$ The absence of target proteins in these cells resembling normal hepatocytes encouraged us to suggest the overexpression of 6D5 ligands in hepatocellular carcinogenesis. Moreover, we also found that Jurkat cells lack

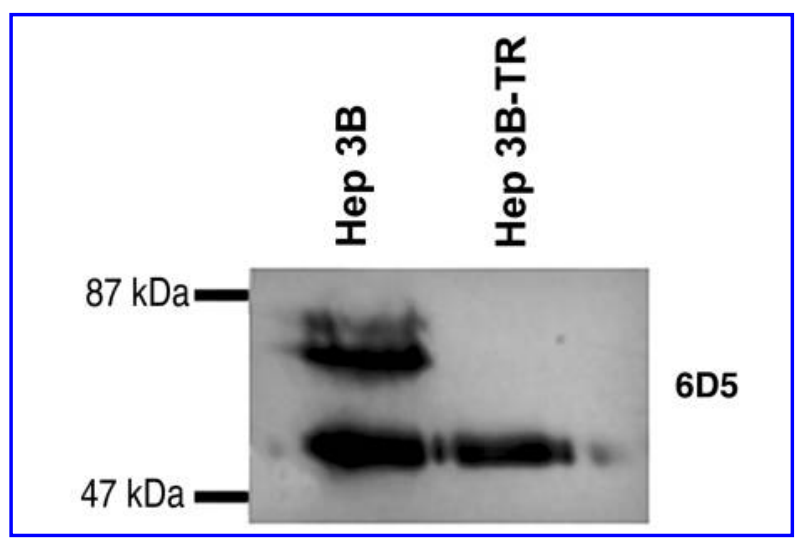

FIG. 5. Secreted forms of 6D5 target proteins. Protein secretion was evaluated in isogenic cell lines Hep3B and Hep3BTR. Proteins were immunoprecipitated from serum-free supernatants of these cells. Following SDS-PAGE and electroblotting, the membrane was probed with 6D5 antibody. Target protein bands were detected only in the supernatant of Hep3B cells. Lower bands appearing in both supernatants correspond to immunoglobulin heavy chain. these proteins and hypothesized that 6D5 ligand expression occurs in a tissue-restricted manner. Taken together, we propose that 6D5 target proteins are underexpressed in normal liver, upregulated in well-differentiated $\mathrm{HCC}$, and attain culmination in poorly differentiated tumors.

The appearance of multiple bands in our Western blots might be due to the expression of isoforms and/or post-translationally modified forms of the 6D5 target protein rather than spontaneous protein degradation, since reprobing the membrane with anti-calnexin antibody revealed single bands in all studied cell lines. This also led us to seek known HCC markers displaying different forms and showing the same pattern as 6D5 ligands in similar studies. The most widely used HCC tumor marker is AFP, which may be translated from multiple RNA transcripts (i.e., $2.2,1.7,1.6$, and $1.35 \mathrm{~kb}$ ), resulting in the expression of protein isoforms with molecular weights similar to those of 6D5 target proteins. ${ }^{(22)}$ However, previous studies have clearly shown that AFP expression is restricted to well-differentiated cell lines and is absent in others. ${ }^{(23,24)}$ Therefore, according to our Western blot experiments showing that 6D5 ligands are expressed in all HCC cell lines regardless of their differentiation state (Fig. 2), we excluded the possibility that 6D5 antibody is directed against AFP epitopes. Another protein, which is subjected to intensive investigation, is glypican-3; results from many studies support its usefulness as a promising marker for HCC. ${ }^{(8,25)}$ This protein too, when expressed, gives rise to several isoforms, whose molecular weights approximate those of protein bands that we observed in our Western blot analyses. However, glypican-3 is a proteoglycan bound to the cell membrane through GPI anchor, whereas no membrane staining was observed on HCC cells in our immunofluorescence assay with 6D5 antibody (Fig. 4). Another group of proteins that displays differential expression in response to cellular stresses and is overexpressed in many cancers consists of members of heat shock proteins (HSP). Most HSPs reside in the cytoplasm and ER and counteract apoptotic stimuli by preventing misfolding of proteins. (26) Two important members, with molecular weights of 70 and $72 \mathrm{kDa}$, respectively, include Hsp70 and GRP78/BiP and have been recently reported to be upregulated in HCC. (27) Their expression was repeatedly shown in Jurkat 


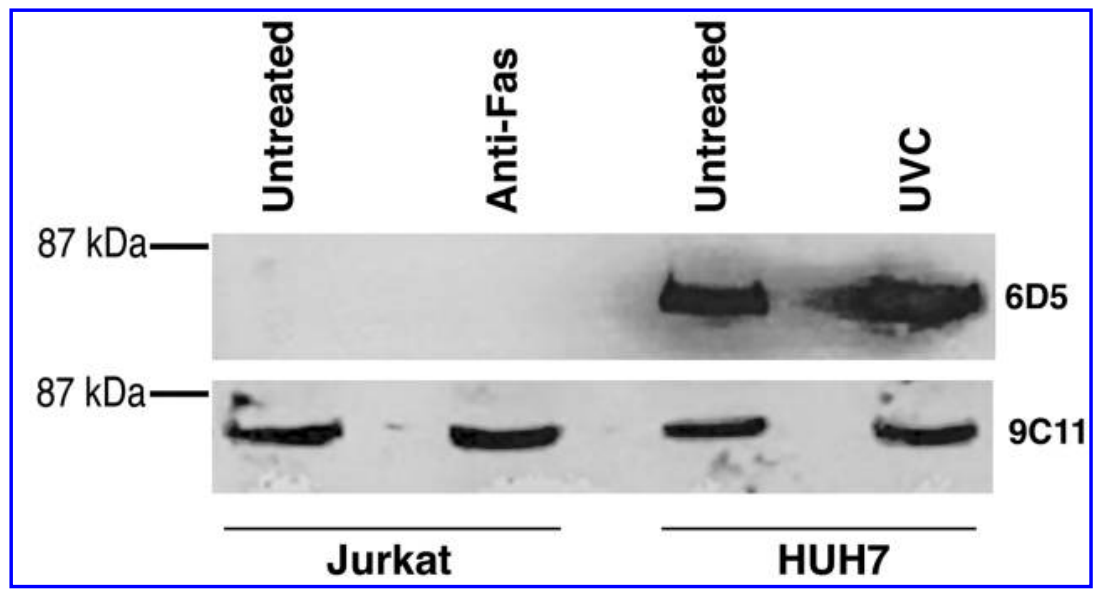

FIG. 6. Induction of apoptosis in HUH7 and Jurkat cells. Apoptosis was induced in HUH7 and Jurkat cells by UVC and antiFas antibody treatment, respectively. Cells were collected at $24 \mathrm{~h}$ post-treatment and analyzed by Western blotting. An induction on 6D5 target protein expression was observed in HUH7 cells upon UVC treatment. However, no protein expression occurred in anti-Fas treated and untreated Jurkat cells (upper row). The membrane was also probed with 9C11 antibody obtained from the same fusion experiment. This antibody recognized proteins in both cell lines in both control and treated cells (lower row). Protein ligands of 9C11 appear not to be modulated upon apoptosis induction.

cells, ${ }^{(28,29)}$ which failed to display 6D5 ligands both in apoptotic and nonapoptotic conditions (Fig. 6). We therefore reasoned that these proteins could not be the targets of our monoclonal antibody.

Immunofluorescence analysis with 6D5 antibody revealed perinuclear and granular staining pattern reminiscent of an endoplasmic reticulum location and prompted us to investigate the secretion of proteins recognized by our monoclonal antibody. However, secreted forms of target proteins were observed only in a limited number of HCC cell lines. Nevertheless, we cannot exclude the possibility that the levels of secreted proteins by other cells might be below the detection limit of our IP-WB. Therefore, it would be useful to screen HCC patients' sera with our antibody to validate the tumor marker potential of target proteins.

Tumor cells harbor mechanisms enabling them to bypass apoptotic cell death, which might result from cellular stresses, and attack by immune effector cells and anti-cancer therapeutic agents. Proteins involved in apoptotic processes are subject to differential modulation upon encounter with genotoxic stimuli. ${ }^{(30)}$ In our study, we triggered apoptosis by treating HUH7 cells with UVC irradiation and examined the expression of 6D5 target proteins. It has been reportedly shown that protein synthesis is inhibited in cells exposed to UV stress through phosphorylation of the $\alpha$ subunit of eukaryotic translation initiation factor $2 .^{(31,32)}$ One of the latter studies also reported a sustained suppression of translation for up to $48 \mathrm{~h} .{ }^{(31)}$ This is in sharp contrast with our observation that 6D5 target proteins display an intermediate-to-late upregulation, which culminates at $24 \mathrm{~h}$ post-UVC treatment.

The gene expression pattern of cells exposed to genotoxic stresses varies enormously depending on both the experimental conditions and genetic backgrounds of cells. ${ }^{(33)}$ This phenomenon supports our results that 6D5 ligands are subject to modulation in our experimental settings based on UVC irradiation. However, taken together with our data showing abundant expression, as well as secretion of target proteins by HCC cell lines, we can conclude that the ligands of our antibody are some of the molecular players involved in liver tumorigenesis. Target protein identification and examination of the reactivity of this antibody with tissues and sera of HCC patients would be useful for the exploitation of 6D5 in diagnostic and prognostic studies of HCC.

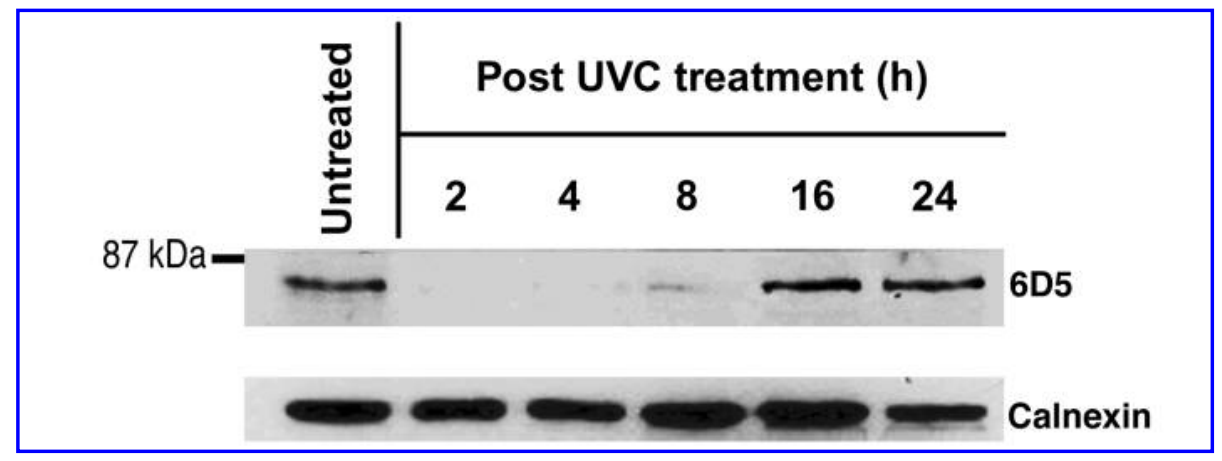

FIG. 7. Intermediate-to-late induction of 6D5 target proteins in apoptotic HUH7 cells. UVC-treated HUH7 cells were collected at given time points and compared with untreated control by Western blotting. Immunoblot was first probed with 6D5 antibody (upper row), and equal protein loading was assessed by reprobing the membrane with anti-calnexin antibody (lower row). 


\section{ACKNOWLEDGMENTS}

This work was supported by grants from the Scientific and Technical Research Council of Turkey (Project 102T075) and Bilkent University Research Funds.

\section{REFERENCES}

1. Bruix J, Boix L, Sala M, and Llovet JM: Focus on hepatocellular carcinoma. Cancer Cell 2004;5:215-219.

2. Llovet JM, Burroughs A, and Bruix J: Hepatocellular carcinoma. Lancet 2003;362:1907-1917.

3. Parkin DM, Bray F, Ferlay J, and Pisani P: Estimating the world cancer burden: GLOBOCAN 2000. Int J Cancer 2001;94:153-156.

4. Bruix J, Barrera JM, Calvet X, et al: Prevalence of antibodies to hepatitis $\mathrm{C}$ virus in Spanish patients with hepatocellular carcinoma and hepatic cirrhosis. Lancet 1989;2:1004-1006.

5. Tsukuma H, Hiyama T, Tanaka S, Nakao M, Yabuuchi T, Kitamura T, Nakanishi K, Fujimoto I, Inoue A, Yamazaki H, and Kawashima T: Risk factors for hepatocellular carcinoma among patients with chronic liver disease. $\mathrm{N}$ Engl J Med 1993;328:1797-1801.

6. Bolondi L: Screening for hepatocellular carcinoma in cirrhosis. J Hepatol 2003;39:1076-1084.

7. Lin DY and Liaw YF: Optimal surveillance of hepatocellular carcinoma in patients with chronic viral hepatitis. J Gastroen Hepatol 2005; 16:715-717.

8. Marrero JA and Fok ASF: Newer markers for hepatocellular carcinoma. Gastroenterology 2004;127:S113-S119.

9. Lopez JB: Recent developments in the first detection of hepatocellular carcinoma. Clin Biochem Rev 2005;26:65-79.

10. Hanahan D and Weinberg RA: The hallmarks of cancer. Cell 2000;100:57-70.

11. Reed JC: Mechanisms of apoptosis avoidance in cancer. Curr Opin Oncol 1999;11:68-75.

12. Soengas MS, Capodieci P, Polsky D, Mora J, Esteller M, OpitzAraya X, McCombie R, Herman JG, Gerald WL, Lazebnik YA, Cordón-Cardó C, and Lowe SW: Inactivation of the apoptosis effector Apaf-1 in malignant melanoma. Nature 2001;409:207-211.

13. Sayan BS, Ince G, Sayan AE, and Ozturk M: NAPO as a novel marker for apoptosis. J Cell Biol 2001;155:719-724.

14. Harlow E and Lane D: Antibodies: A Laboratory Manual. Cold Spring Harbor Laboratory Press, Cold Spring Harbor, NY, 1988. pp. 196-214.

15. Bradford MM: A rapid and sensitive method for the quantitation of microgram quantities of protein utilizing the principle of protein-dye binding. Anal Biochem 1976;72:248-254.

16. Inagaki M, Moustakas A, Lin HY, Lodish HF, and Carr BI: Growth inhibition by transforming growth factor $\beta$ (TGF- $\beta$ ) type I is restored in TGF- $\beta$-resistant hepatoma cells after expression of TGF- $\beta$ receptor type II cDNA. Proc Natl Acad Sci USA 1993;90:5359-5363.

17. Arad U, Axelrod J, Ben-nun-Shaul O, Oppenheim A, and Galun E: Hepatitis B virus enhances transduction of human hepatocytes by SV40-based vectors. J Hepatol 2004;40:520-526.

18. Liu Y, Takahashi S, Ogasawara H, Seo HG, Kawagoe M, Hirasawa F, Guo N, Ueno Y, Kameda T, and Sugiyama T: Protection of hepatocytes from apoptosis by a novel substance from actinomycetes culture medium. Biomed Res 2005;26:9-14.

19. Ozturk N, Erdal E, Mumcuoglu M, Akcali KC, Yalcin O, Senturk S, Arslan-Ergul A, Gur B, Yulug I, Cetin-Atalay R, Yakicier C, Yagci T, Tez M, and Ozturk M: Reprogramming of replicative senescence in hepatocellular carcinoma-derived cells. Proc Natl Acad Sci USA 2006;103:2178-2183.
20. Nagamori S, Hasumura S, Matsuura T, Aizaki H, and Kawada M: Developments in bioartificial liver research: concepts, performance, and applications. J Gastroenterol 2000;35:493-503.

21. Aizaki H, Nagamori S, Matsuda M, Kawakami H, Hashimoto O, Ishiko $\mathrm{H}$, Kawada M, Matsuura T, Hasumura S, Matsuura $\mathrm{Y}$, Suzuki T, and Miyamura T: Production and release of infectious hepatitis $\mathrm{C}$ virus from human liver cell cultures in the three-dimensional radial-flow bioreactor. Virology 2003;314:16-25.

22. Mizejewski GJ: Alpha-fetoprotein structure and function: relevance to isoforms, epitopes, and conformational variants. Exp Biol Med 2001;226:377-408.

23. Lee JS and Thorgeirsson SS: Functional and genomic implications of global gene expression profiles in cell lines from human hepatocellular cancer. Hepatology 2002;35:1134-1143.

24. Kawai HF, Kanaeko S, Honda M, Shirota Y, and Kobayashi K: $\alpha$ fetoprotein-producing hepatoma cell lines share common expression profiles of genes in various categories demonstrated by cDNA microarray analysis. Hepatology 2001;33:676-691.

25. Nakatsura T, Yoshitake Y, Senju S, Monji M, Komori H, Motomura Y, Hosaka S, Beppu T, Ishiko T, Kamohara H, Ashihara H, Katagiri T, Furukawa Y, Fujiyama S, Ogawa M, Nakamura Y, and Nishimura Y: Glypican-3, overexpressed specifically in human hepatocellular carcinoma, is a novel tumor marker. Biochem Bioph Res Co 2003;306:16-25.

26. Hendrick JP and Hartl F: Molecular chaperone functions of heatshock proteins. Ann Rev Biochem 1993;62:349-384.

27. Luk JM, Lam CT, Siu AFM, Lam BY, Ng IOL, Hu MY, Che CM, and Fan ST: Proteomic profiling of hepatocellular carcinoma in Chinese cohort reveals heat-shock proteins (Hsp27, Hsp70, GRP78) up-regulation and their associated prognostic values. Proteomics 2006;6:1049-1057.

28. Lewis ML and Hughes-Fulford M: Regulation of heat shock protein message in Jurkat cells cultured under serum-starved and gravity-altered conditions. J Cell Biochem 2000;77:127-134.

29. Kuwabara H, Yoneda M, Hayasaki H, Nakamura T, and Mori H: Glucose regulated proteins 78 and 75 bind to the receptor for hyaluronan mediated motility in interphase microtubules. Biochem Bioph Res Co 2006;339:971-976.

30. McKay BC, Stubbert LJ, Fowler CC, Smith JM, Cardamore RA, and Spronck JC: Regulation of ultraviolet light-induced gene expression by gene size PNAS 2004;101:6582-6586.

31. Wu S, Hu Y, Wang JL, Chatterjee M, Shi Y, and Kaufman RJ: Ultraviolet light inhibits translation through activation of the unfolded protein response kinase PERK in the lumen of the endoplasmic reticulum. J Biol Chem 2002;277:18077-18083.

32. Deng J, Harding HP, Raught B, Gingras AC, Berlanga JJ, Scheuner D, Kaufman RJ, Ron D, and Sonenberg N: Activation of GCN2 in UV-irradiated cells inhibits translation. Curr Biol 2002;12:1279-1286.

33. Koch-Paiz CA, Amundson SA, Bittner ML, Meltzer PS, Fornace AJ Jr: Functional genomics of UV radiation responses in human cells. Mutat Res 2004;549:65-78.

Address reprint requests to: Tamer Yagci, M.D., Ph.D. Bilkent University Faculty of Science Department of Molecular Biology and Genetics 06800, Bilkent-Ankara Turkey

E-mail: tyagci@fen.bilkent.edu.tr 
This article has been cited by:

1. Gorkem Odabas, Metin Cetin, Serdar Turhal, Huseyin Baloglu, A. Emre Sayan, Tamer Yagci. 2018. Plexin C1 Marks Liver Cancer Cells with Epithelial Phenotype and Is Overexpressed in Hepatocellular Carcinoma. Canadian Journal of Gastroenterology and Hepatology 2018, 1-9. [Crossref]

2. Emin Oztas, M. Ender Avci, Ayhan Ozcan, A. Emre Sayan, Eugene Tulchinsky, Tamer Yagci. 2010. Novel monoclonal antibodies detect Smad-interacting protein 1 (SIP1) in the cytoplasm of human cells from multiple tumor tissue arrays. Experimental and Molecular Pathology 89:2, 182-189. [Crossref]

3. Mehmet Ender Avci, Ozlen Konu, Tamer Yagci. 2008. Quantification of SLIT-ROBO transcripts in hepatocellular carcinoma reveals two groups of genes with coordinate expression. BMC Cancer 8:1. . [Crossref] 